\title{
Current Practices on Diagnosis and Management of Women With Vulvodynia
}

\author{
Marcela Grigol Bardin ( $\sim$ bardinmarcela@gmail.com ) \\ State University of Campinas \\ Paulo César Giraldo \\ State University of Campinas \\ Júlia Ferreira Fante \\ State University of Campinas \\ Camila Carvalho de Araujo \\ State University of Campinas \\ Marie-Pierre Cyr \\ Faculty of Medicine and Health Sciences, Sherbrooke \\ Andrea de Andrade Marques \\ State University of Campinas
}

\section{Research Article}

Keywords: Diagnosis, Pain clinics, Pain management, Vulvodynia, Vestibulodynia

Posted Date: February 26th, 2021

DOl: https://doi.org/10.21203/rs.3.rs-247137/v1

License: (9) This work is licensed under a Creative Commons Attribution 4.0 International License. Read Full License 


\section{Abstract \\ Introduction and hypothesis}

: To describe clinical characteristics, previous medical assessment, past treatments and vulvar pain relief among women with vulvodynia.

\section{Methods}

Brazilian women with vulvodynia $(n=144)$ were assessed for vaginal infection and vulvar pain intensity by means of a cotton swab test based on a numerical rate scale (NRS). All women answered the Female Sexual Function Index questionnaire and a structured instrument about present vulvar symptoms and previously experienced treatments. Vulvar pain relief achieved with previous treatments was qualified through a 4-point Likert-scale.

\section{Results}

Previous vulvar pain duration was $5.8( \pm 4)$ years. More than $50 \%$ consulted with three or more physicians and $49 \%$ remained without a conclusive diagnosis. Diagnosis and treatment vulvovaginal infection was very common.

The most commonly used treatments were lubricants (66\%), topical anesthetics (36\%) and vulvar care techniques (36\%). All of then provided only low pain relief.

Physical therapy and oral gabapentin provided strong vulvar pain relief.

\section{Conclusion}

Prolonged duration of vulvar pain, multiple visits to healthcare professionals and poor relief of pain are common aspects in the clinical history of women with vulvodynia. Vulvovaginal symptoms other than pain are common, highlighting the importance of the screening tests in order to avoid misdiagnosis.

\section{Introduction}

Vulvar pain is a highly prevalent symptom among women of all ages [1]. Many causes may lead to vulvar pain or discomfort, such as vaginal or urinary infection, neurologic disorder, local trauma, hormone imbalance, immunosuppressive diseases, among others [2]. However, the diagnosis of vulvodynia is characterized by chronic vulvar pain or irritation that occurs without a clear identifiable cause [2].

The prevalence of vulvodynia is relatively high, affecting from 10 to $28 \%$ of women in the United States $[1,3,4]$. However, this number may still underestimate the real prevalence of the condition, since previous studies report vulvodynia as an underdiagnosed condition $[1,3,5]$. The possible reason why the diagnosis of vulvodynia remains a challenge for both patients and practitioners may be due to unawareness of the condition and lack of infrastructure required in the diagnosis exclusion process. Furthermore, it is important that health care professionals recognize the many subtypes of vulvodynia, in order to understand its broad manifestations. Vulvodynia can be 1) localized or generalized; 2) provoked, unprovoked or mixed; and 3) primary or secondary, depending on whether vulvar pain started at the beginning of sexual activity or sometime after painless intercourse, respectively [2]. In addition, pain can be described in a different manner by patients, who usually use words such as "burning", "itching", "stinging", "irritation", "stabbing" and/or "rawness" [1]. Such variation when reporting symptoms to health care providers might lead to a delay in diagnosis or even misdiagnosis and thus lead to unsuitable treatment [1].

Owing to the unclear pathophysiology and multifactorial characteristic of vulvodynia, several potential treatment modalities are used to reduce pain intensity and/or improve sexual function [6, 7]. According to practice guidelines, most common available modalities are vulvar care (genital hygiene and clothing recommendations); psychotherapy; oral analgesics; topical anaesthetics; 
tricyclic antidepressants; anticonvulsants and physical therapy of the pelvic floor muscles [5, 6-8]. However, there is no gold standard treatment for vulvodynia, and there is a lack of knowledge of whether those modalities promote long-term relief of vulvar pain. The success of treatment in obtaining relief from symptoms in women with vulvodynia may depend on correct diagnosis and adherence to recommended treatment.

There seems to be a lack of awareness of adequate diagnosis for women with this debilitating condition during routine gynecologic consultations [4, 9] misdiagnosis of vulvodynia may lead to multiple attempts at treatment, increasing expenses both for the individual and society $[5,10]$. Moreover, anxiety, psychological burden and sexual dysfunction may be consequences of a misdiagnosis or delayed diagnosis [11-13]. Despite that, no studies have investigated the course of patients seeking health care since the onset of vulvar symptoms in the Brazilian population. This type of information is essential for the development of strategies to improve accuracy of the diagnosis and treatment of vulvodynia. The knowledge seeking care process for vulvar pain diagnosis and treatment should be pursued and improved.

The aim of the present study is to explore clinical characteristics, previous diagnosis, current vulvovaginal signs and symptoms, pain intensity, sexual function, as well as self-reported vulvar pain relief, obtained by previous treatment experienced in women suffering from vulvodynia.

\section{Methods}

This is a descriptive study, part of a larger randomized study, however, the data presented here are previous to the randomization. The aim is to describe clinical characteristics, medical assessment, treatments and vulvar pain relief among women with vulvodynia. This is a cross sectional descriptive study comprised of data derived from a larger, randomized stud, can be accessed in Clinicaltrials.gov, registration number NCT02871661.

The study was approved by the Institutional Review Board of the University of Campinas, under number 1.588.648, CAAE: 54665316.5.0000.5404.

Women were invited to participate in the study when seeking the Family Planning (12\%), Genital Infection Disease (14\%) or Pelvic Rehabilitation (20\%) Outpatient Clinic of the Women's Hospital of the Campinas University, and through local radio and television calls (54\%).

Patients interested in volunteering were asked to contact the main researcher and set a pre-screening interview in order to verify eligibility criteria.

There were 167 premenopausal women recruited, aged 18 years of age or older who reported having vulvar pain lasting for at least three months. The diagnosis of vulvodynia was confirmed at the evaluation assessment by either, complaint of spontaneously or provoked vulvar pain (during or after intercourse) for more than three months [14] or based on Friedrich criteria modified by Bergeron et al and described elsewhere [15]. Therefore, all subtypes of vulvodynia were included.

Additional informed consent was obtained from all individual participants for whom identifying information is included in this article. All volunteers underwent a physical examination performed by a gynecologist to exclude other possible diagnoses associated with vulvar pain, with vaginal content collection for bacterioscopy and fungal growth analysis in laboratory. Vaginal $\mathrm{pH}$ was also measured using an indicator paper ( $\mathrm{pH}$ indicator strips; Merck Laboratories, Germany) placed on the right side of the vagina wall for one minute.

Exclusion criteria included history of neoplasm, cognitive alteration, pregnancy, breastfeeding, diagnosed premature ovarian insufficiency, previous oophorectomy, anterior and/or posterior surgical repair and vulvovaginal candidiasis or bacterial vaginosis, and atrophic vaginitis. Women with persistent vulvar pain after treatment were included in the study.

All volunteers signed an informed consent form.

After the eligibility criteria was confirmed, volunteers completed out a structured interview about sociodemographic, medical and gynecological history. Current vulvovaginal symptoms were assessed by physical examination, searching for vulvar fissure, 
oedema and erythema, vaginal discharge, and foul odor. Pain was elicited in response to light pressure using the cotton swab test (CST). Mean score of sexual pain was based on the last 30 days and pain intensity was based on a numerical rating scale (NRS). Patients were asked to choose a number from 0 (no pain) to 10 (worst pain) that best represented pain intensity. In this study, a trained physical therapist applied gentle pressure in the following regions: $3 \mathrm{~h}, 4 \mathrm{~h}-5 \mathrm{~h}, 6 \mathrm{~h}, 7 \mathrm{~h}-8 \mathrm{and} 12 \mathrm{~h}$ positions of the vaginal clock using a sterile cotton swab, and asked the patient to immediately rate pain sensation after pressure was applied to each position. The CST reported in this study was averaged considering all positions tested. Vulvar pain on attempts of vaginal penetration, during or after sexual intercourse, was self-reported by patients, and considered the main discomfort attributed to sexual intercourse.

For assessment of sexual function, women completed a self-reported questionnaire on sexual function, the Female Sexual Function Index (FSFI) [16]. This 19-item self-report questionnaire assesses global sexual functioning, including specific evaluations of desire, arousal, lubrication, orgasm, sexual satisfaction, and pain. Scores ranged from to 2 to 36 . Women with a score equal to or less than 26 were identified as having sexual dysfunction, as suggested by scientific medical literature [16].

The patients answered a questionnaire that included all the treatment options for vulvodynia in order to inform about previous treatments. They were requested to mark treatment modes used specifically to manage vulvodynia.

Symptom relief of vulvar and sexual pains were reported according to a 4-point Likert-Scale, based on NRS.

Regression of 0 point on the NRS was compatible to "no relief"; 1 point of regression, "poor relief"; 2 points of regression on the NRS, "mild relief" and 3 or more points of regression on NRS "strong relief of vulvar pain". The scale had a Cronbach's Alpha of a $=.871$, suggesting high internal consistency.

Statistical analysis used descriptive methods. Data information presenting categorical variables are shown as frequency (n) and percentage (\%). Continuous data are presented as mean and standard deviation. In order to increase accuracy when measuring sexual function of the population studied, women who reported not having current sexual intercourse were analysed as missing data for FSFI, so the total score would not underestimate the sexual function of this sample, as proposed by Jiann, 2012 [17].

\section{Results}

To identify vulvodynia cases, 169 women were screened, 144 were eligible for the study (Fig. 1). Mean patient age was 29 ( \pm 9.2 ) years old. Population characteristics of participants are shown in Table 1. 
Table 1

Sociodemographic characteristics of women with vulvodynia enrolled in the study $(n=144)$

\begin{tabular}{|c|c|}
\hline Variables & Frequency $\mathrm{n}(\%)$ \\
\hline Age (years, mean \pm SD) & $29( \pm 9.2)$ \\
\hline Caucasian ethinicity & 105 (73\%) \\
\hline Education & $22(15.3 \%)$ \\
\hline High school & $96(67 \%)$ \\
\hline College or undergraduate degree & $26(18.7 \%)$ \\
\hline \multicolumn{2}{|l|}{ Graduate school or professional training } \\
\hline Religion & $68(46.5 \%)$ \\
\hline Catholic & $43(30.3 \%)$ \\
\hline Other & $33(23.2 \%)$ \\
\hline \multicolumn{2}{|l|}{ None or atheist } \\
\hline BMI (mean \pm SD) & $22.6( \pm 4.8)$ \\
\hline Relationship status & $13(9.2 \%)$ \\
\hline No partner & $5(3.3 \%)$ \\
\hline Sporadic partner & $126(87.5 \%)$ \\
\hline \multicolumn{2}{|l|}{ Steady partner } \\
\hline Parity & $100(69.4 \%)$ \\
\hline Nulliparous & $14(9.7 \%)$ \\
\hline 1 & $30(20.9 \%)$ \\
\hline \multicolumn{2}{|l|}{2 or more } \\
\hline Miscarriage & $10(6.9 \%)$ \\
\hline \multicolumn{2}{|l|}{ Yes } \\
\hline Deliveries & $110(76.4 \%)$ \\
\hline None & $22(15.3 \%)$ \\
\hline Caesarion section & $12(8.3 \%)$ \\
\hline \multicolumn{2}{|l|}{ Vaginal birth } \\
\hline Previous pelvic surgery (other than cesarian) & $21.5(22.9 \%)$ \\
\hline \multicolumn{2}{|l|}{ Yes } \\
\hline Sedentary lifestyle & $58(40.3 \%)$ \\
\hline \multicolumn{2}{|l|}{ Yes } \\
\hline Associated health problem & $55(38.2 \%)$ \\
\hline Yes & \\
\hline
\end{tabular}




\begin{tabular}{|ll|}
\hline Variables & Frequency $\mathbf{n}(\%)$ \\
\hline Contraception method* & $2(1.4 \%)$ \\
Hone & $61(41.3 \%)$ \\
Condom & $33(23 \%)$ \\
Other & $(a)$ \\
\hline Tabaco use & $60(14.1 \%)$ \\
Yes & \\
\hline
\end{tabular}

Comorbid conditions were common and found in $38 \%(n=55)$ of the studied population: $19.4 \%$ had been previously diagnosed with either depression or anxiety disorder, of whom 16 patients were taking antidepressants; $9.7 \%$ had a previous diagnosis or clinical suspicion of endometriosis; $1.4 \%$ had lupus; $4.9 \%$ had bladder pain syndrome; $2 \%$ had irritable bowel syndrome and $2.8 \%$ had fibromyalgia. More than one comorbid condition was found in $19 \%$.

History of vulvar pain lasted from five months to 19 years, with a mean of 5.8 ( \pm 4.1 SD) years. The mean time interval between the onset of symptoms and diagnosis of vulvodynia was 5 ( \pm 4.3 SD) years. Only $29.4 \%$ had a confirmed diagnosis of vulvodynia prior to participating in the present study $(n=40)$, and the mean time elapsed since detection of vulvodynia was 2.6 $( \pm 8.6 \mathrm{SD})$ years.

Vulvar signs and symptoms reported, in addition to or instead of pain, were: burning (81.2\%), erythema (57.6\%) and itching (36.1\%), see Table 2.

Most women (82.4\%) had sought medical help, and $50.7 \%$ had visited three or more health care professionals with vulvar pain as the main reason for consultation.

However, $49.2 \%$ of these patients remained without any diagnosis, and $21.3 \%$ received a diagnosis other than vulvodynia as the cause of vulvar pain (Table 2).

One-third of the participants reported at least one treatment for vaginal infection in the last 12 months.

Of those, $89 \%$ informed that the infection was candidiasis. However, only $28 \%$ were tested for vaginal infection through laboratory exams. 
Table 2

Clinical characteristics of women with vulvodynia $(n=144)$

\begin{tabular}{|c|c|}
\hline Vulvovaginal signs and symptoms* & $83(57.6 \%)$ \\
\hline Vulvar erythema & $80(55.5 \%)$ \\
\hline Current vaginal discharge & $37(25.7 \%)$ \\
\hline Bad odor & $30(20.8 \%)$ \\
\hline Fissure & $27(18.7 \%)$ \\
\hline Oedema & $117(81.2 \%)$ \\
\hline $\begin{array}{l}\text { Current vulva/vaginal burning } \\
\text { Itching }\end{array}$ & $52(36.1 \%)$ \\
\hline Patients seeking health care professionals $\left(n=136^{* \star}\right)$ & $24(17.6 \%)$ \\
\hline None & $21(15.5 \%)$ \\
\hline 1 & $22(16.2 \%)$ \\
\hline $\begin{array}{l}2 \\
3 \text { or more }\end{array}$ & $69(50.7 \%)$ \\
\hline Type of health professional visited* $(n=136)$ & $28(17.6 \%)$ \\
\hline None & $104(76.5 \%)$ \\
\hline Gynecologist & $32(23.5 \%)$ \\
\hline Psychiatrist & $11(8.2 \%)$ \\
\hline $\begin{array}{l}\text { Physical therapist } \\
\text { Others }^{(a)}\end{array}$ & $7(5.2 \%)$ \\
\hline Previous Diagnosis** $(n=136)$ & $67(49.2 \%)$ \\
\hline None & $5(3.7 \%)$ \\
\hline Low estrogen & $21(15.5 \%)$ \\
\hline Candidiasis & $3(2.2 \%)$ \\
\hline $\begin{array}{l}\text { Non-organic }{ }^{(b)} \\
\text { Vulvodynia }\end{array}$ & $40(29.4 \%)$ \\
\hline Vulvodynia setting & $61(42.4 \%)$ \\
\hline $\begin{array}{l}\text { Primary } \\
\text { Secondary }\end{array}$ & $83(57.6 \%)$ \\
\hline Vulvodynia subtype & $106(73.6 \%)$ \\
\hline Localized provoked & $8(5.5 \%)$ \\
\hline Localized mixed & $12(8.4 \%)$ \\
\hline $\begin{array}{l}\text { Generalized provoked } \\
\text { Generalized mixed }\end{array}$ & $18(12.5 \%)$ \\
\hline CST test according to NRS (mean \pm SD) & $5.1( \pm 3.3)$ \\
\hline
\end{tabular}




\begin{tabular}{|ll|}
\hline Sexual pain on last 30 days (mean \pm SD, $\mathrm{n}=132)$ & $7.06( \pm 2.9)$ \\
\hline FSFI total score $(\mathrm{N}=126)$ & $118(93.6 \%)$ \\
\hline $\mathbf{2 6 . 5 5}$ & \\
\hline
\end{tabular}

Secondary (57.6\%) and localized provoked (73.6\%) were the most prevalent subtypes of vulvodynia. Self-reported average pain score during the last sexual intercourse was high, with a mean score for FSFI of 16.8 ( $\pm 7.7 \mathrm{SD})$. No sexual intercourse in the last 30 days was reported by 18 (12.5\%) women, of whom five said being due to pain reasons.

More than $20 \%(n=34)$ did not undergo any treatment for vulvodynia. Among treated women, most had tried at least two $(2 \pm 1.6$ SD) treatment options to deal with vulvar pain, and the majority described poor relief or no relief. The most commonly cited treatments for "strong relief of vulvar pain" (three or more decreased points in pain according to NRS) were physical therapy for the pelvic floor muscles, psychotherapy, tricyclic antidepressant, gabapentin and topical anesthetics (Table 3).

Use of lubricant during intercourse (67.5\%) and specific hygiene/vulvar care (34.2\%) were also frequently reported as techniques adopted to reduce vulvar pain.

Other treatments used were topical steroid, antifungal, low oxalate diet and other antidepressants.

Table 3

Vulvar pain relief reported in previous treatment for vulvodynia

\begin{tabular}{|lllll|}
\hline Treatment or method used $(\mathbf{n = 1 1 0 )}$ & Vulvar pain relief & & \\
\hline (N-\%) & No relief & Low & Mild & Strong \\
& $\mathbf{n}(\%)$ & $\mathbf{n}(\%)$ & $\mathbf{n}(\%)$ & $\mathbf{n}(\%)$ \\
\hline Lubricant (73-66.4\%) & $43(58.9)$ & $23(31.5)$ & $5(6.8)$ & $2(2.8)$ \\
\hline Topical anesthetic (40-36.4\%) & $4(10)$ & $21(52.5)$ & $10(25)$ & $5(12.5)$ \\
\hline Specific hygiene (37-33.6\%) & $26(70.3)$ & $8(21.6)$ & $3(8.1)$ & 0 \\
\hline Oral Antifungal (36-32.7\%) & $27(75)$ & $8(22.2)$ & $1(2.8)$ & - \\
\hline Oral Tricyclic antidepressant (34-31\%) & $11(32.3)$ & $16(47)$ & $5(14.7)$ & $2(6)$ \\
\hline Topical antifungal (28-25.5\%) & $25(89.2)$ & $1(3.6)$ & $1(3.6)$ & $1(3.6)$ \\
\hline Physical therapy for pelvic floor muscles (18-16.4\%) & $2(11.1)$ & $2(11.1)$ & $8(44.4)$ & $6(33.4)$ \\
\hline Psychotherapy approach (17-15.5\%) & $7(41.2)$ & $4(23.5)$ & $4(23.5)$ & $2(11.8)$ \\
\hline Other antidepressant (14- 13\%) & $9(64.3)$ & $5(35.7)$ & - & - \\
\hline Topical hormone (11 - 10\%) & $3(27.3)$ & $5(45.4)$ & $2(18.2)$ & $1(9.1)$ \\
\hline Oral hormone therapy (8- 7\%) & $6(75)$ & $1(12.5)$ & $1(12.5)$ & - \\
\hline Oral Gabapentin (6-5.2\%) & $2(33.3)$ & $2(33.3)$ & $1(16.6)$ & $1(16.6)$ \\
\hline Topical gabapentine (5-4.5\%) & $1(20)$ & $2(40)$ & $2(40)$ & 0 \\
\hline Low oxalate diet (5-4.3\%) & $3(60)$ & 0 & $1(20)$ & $1(20)$ \\
\hline Topical amitriptyline (3-2.6\%) & $2(66.7)$ & 0 & $1(33.3)$ & 0 \\
\hline *More than one treatment was possible & & & & \\
\hline Declarations & & & & \\
\hline
\end{tabular}




\section{Discussion}

Our data showed a high number of women who had years of lasting of vulvar pain, mostly who sought three or more doctors and either remained without diagnosis or received diagnosis different to vulvodynia. As a consequence of the probable inappropriate diagnosis received for the vulvar pain complaint, these women were frequently treated with inappropriate therapies for the management of vulvodynia, such as antifungal and topical hormones.

Some of these findings are in line with the results showed by Harlow \& Stewart's [3], that two-thirds of North-American women with chronic vulvar pain who sought medical care visited three or more physicians, many of whom failed to provide a diagnosis. Scientific literature suggests lack of awareness of the condition by both practitioners and patients as the main reason for the low rates of correct diagnosis and appropriate treatment $[1,15]$. The present study also points to the fact that, in addition to typical vulvar pain, other vulvar signs (fissure, vaginal discharge, foul odor, erythema) and symptoms (burning, itching) were frequent. All those characteristics can make the diagnosis unclear, leading to a wrong interpretation of vaginal infection, for instance.

In fact, our data showed that many of the participants who sought medical care had been repeatedly treated for vulvovaginitis, obtaining poor or no symptom relief.

This finding is consistent with Reed et al [1], who found that more than one-third of women diagnosed with vulvodynia were treated for candidiasis before receiving the final diagnosis and presented no improvement in vulvar symptoms. A possible explanation for the large number of women treated for candidiasis before reaching the final diagnosis also relies on the pathogenesis of vulvodynia that associates a high prevalence of yeast infections preceding VVD diagnosis [18]. However, it is important to consider that a large number of women might be diagnosed with candidiasis when complaining of vulvodynia symptoms. This concept has been previously suggested by Harlow et al [19], who affirmed that the diagnosis of vulvodynia and recurrent yeast infection probably have a bidirectional association. In the present study, most participants treated for candidiasis after seeking medical care for the complaint of vulvar pain, reported they had no laboratory tests to confirm the suspicion of infection, and remained with the main symptoms. This finding also suggests that patients are overdiagnosed with vaginal infections when seeking a diagnosis for vulvodynia, supporting the bidirectional association.

Indeed, the opposite might also occur. Our study found that only one-third of the volunteers diagnosed with vulvovaginal disorders at the eligibility screening process still fitted the diagnosis of vulvodynia after treatment. This reinforces the need to follow recommendations for the exclusion of different diagnoses in order to increase accuracy in the diagnosis of vulvodynia [14]. Laboratory tests confirming the hypothesized diagnosis of vaginal infection, for instance, should be encouraged as an objective measure to avoid misdiagnosis.

Furthermore, our data showed that the self-reported average pain score during the last sexual intercourse according to NRS was two points higher than the mean obtained in the CST.

Although our sample was made up of both provoked and unprovoked vulvodynia, the first subtype was highly predominant. Diagnosis criteria for vulvodynia usually follows the ones proposed by Friedrich [20] that include 1) severe pain on vestibular touch or attempted vaginal entry, 2) tenderness to pressure localized within the vulvar vestibule, and 3) physical findings confined to vestibular erythema of various degrees. However, it is important to highlight that although the CST has been broadly used as a reliable tool to confirm the 2 nd criteria, it only predicts provoked vulvodynia subtype. Therefore, self-reported complaints of high intensity of vulvar and/or sexual pain, should enrich clinical guidance for vulvodynia diagnosis as much as CST. Furthermore, Bergeron et al increased generality of vulvodynia diagnosis by showing the reliability of these criteria, modifying Friedrich criteria by limiting it to the 1st and 2nd criteria only, excluding the need to confirm erythema findings on the vulva vestibule [15]. However, more than half of the present studied population was evaluated with vulvar erythema. These findings suggest that although, as proposed by Bergeron et al [15], not all women with vulvodynia may present this clinical sign, it is indeed important that practitioners pay attention to the fact that the presence of vulvar erythema is an expected finding in women with this condition.

In addition to diagnostic issues, a consensus also exists over the difficulty in treating vulvodynia [21]. The most commonly prescribed medication for the treatment of vulvodynia is tricyclic antidepressants, selective norepinephrine reuptake inhibitors and anticonvulsants. Nevertheless, there is no general agreement that any of these drugs is highly effective in relieving vulvar 
symptoms [8]. While some studies suggest that the use of these medications can only benefit women with generalized, unprovoked vulvodynia subtype [22, 23], others have shown similar effects of oral medication comparing the different subtypes of vulvodynia $[7,8,24,25]$. Our data showed that the tricyclic antidepressant was the most widely prescribed drug, but only a small portion of women reported having strong vulvar pain relief. However, further studies are needed to suggest this finding as related to the predominant characteristic of the sample that had the provoked vulvodynia subtype.

A recent meta-analysis comparing medication versus placebo for treatment of vulvodynia showed that both improve sexual function, although medication has a minimally superior effect [26].The same study highlights the importance of adding a more efficacious treatment for female sexual dysfunction. Our data showed that, unfortunately, only a minimum amount of the women assessed received appropriate treatment for the management of vulvodynia.

Nevertheless, the most commonly reported measures were either oral or topical medication (antifungal, hormone, gabapentin, steroids, amitriptyline), followed by lubricants, topical anesthetics and specific hygiene or vulvar care techniques, described as resulting only in a low symptom relief.

Very few women reported having tried other efficacious treatment modes, such as physical therapy, even though it has been recommended by many guidelines as a first-line treatment for vulvodynia [7, 14, 27]. Although only a few women underwent physical therapy to treat vulvodynia in this study, the high percentage of those that reported achieving a high relief of vulvar pain with this approach supports its effectiveness.

Due to the study design, it was not possible to assess whether previous treatment improved sexual function. It is assumed, however, that they would also be characterized as having sexual dysfunction at that time, as this is an expected aspect of women with vulvodynia [4, 9]. Only $10 \%$ of our volunteers scored above the cut-off point, which is indicative of normal sexual function. It is clear that sexual dysfunction in this population is a consequence of vulvar pain, especially during intercourse [11, 28]. Nevertheless, whether the volunteers assessed in this study maintained or worsened their sexual function index over time remains unclear. The fact that this study depicts white, highly educated women, which may not be highly representative of our country, is also a limitation of our study. It is possible that increasing the number of women with vulvodynia, the numbers of delays in achieving a correct diagnosis and appropriate treatment would have increased even more in a population with lower levels of education. In addition, the self-reported characteristic of the study was the basis for the main variables assessed including: number of physicians sought, previous diagnosis and prescribed treatment, level of vulvar discomfort relief, among others. However, previous research has demonstrated a broad agreement between self-reported and medical record data among highly educated women [29]. All women were clinically examined to confirm the diagnosis of vulvodynia, which also provides support to the goals analyzed.

Our findings highlight the importance of developing strategies to increase awareness about the diagnosis of vulvodynia and develop suitable treatment for the condition. These data emphasize the lack of a clear diagnosis of vulvodynia, confirmed by few appropriate treatment modes and a wide use of inefficient methods to deal with persistent vulvar pain.

In summary, a prolonged duration of vulvar pain, multiple visits to healthcare professionals and poor relief of pain with previously undergone treatments are common aspects in the clinical history of women with vulvodynia. Vulvovaginal signs and symptoms other than pain are common, and highlight the importance of having a better clinical and/or laboratorial tests supporting its diagnosis.

\section{Declarations}

Ethics approval and consent to participate: This study has been approved by the Institutional Review Board of the University of Campinas (CAAE: 54665316.5.0000.5404), and written informed consent was obtained from all individual participants included in the study and confidentiality and anonymity were ensured. All procedures performed in studies were in accordance with the ethical standards of the institutional and/or national research committee and with the Helsinki declaration.

Consent for publication: Not applicable. 
Availability of data and materials: The dataset used and analyzed during the current study are available from the corresponding authors upon making official request.

Competing interests: The authors declare that they have no confict of interest.

Funding: We are grateful to Coordenação de Aperfeiçoamento Pessoal de Nível Superior- CAPES for the support of this study with a Post-Graduate Scholarship to one of the authors (MB), and Santander Mobilidade Internacional also financed her Internship to the University of Sherbrooke, Canada, where this article was written and revised. The funders had no role in conducting the research or reporting the fundings.

Authors' contributions: MG Bardin: Project development, Data collection, Data analysis, Manuscript writing and editing. PC Giraldo: Project development, Data analysis, Manuscript writing and editing. JF Fante: Data collection. CC Araujo: Data analysis, Manuscript editing and editing. MP Cyr: Data analysis, Manuscript editing and editing. AA Marques: Data analysis, Manuscript editing and editing.

All authors read and approved the final manuscript.

Acknowledgements: The authors thank all patients that have participated in this study.

\section{Authors' information (optional)}

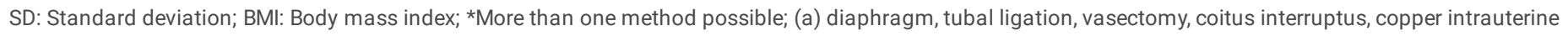
device.

: Not applicable.

\section{References}

1. Reed, B.D., et al., Prevalence and demographic characteristics of vulvodynia in a population-based sample. Am J Obstet Gynecol, 2012. 206(2): p. 170 e1-9.

2. Bornstein, J., et al., 2015 ISSVD, ISSWSH, and IPPS Consensus Terminology and Classification of Persistent Vulvar Pain and Vulvodynia. J Sex Med, 2016. 13(4): p. 607-12.

3. Harlow, B.L. and E.G. Stewart, A population-based assessment of chronic unexplained vulvar pain: have we underestimated the prevalence of vulvodynia? J Am Med Womens Assoc (1972), 2003. 58(2): p. 82-8.

4. Arnold, L.D., et al., Assessment of vulvodynia symptoms in a sample of US women: a prevalence survey with a nested case control study. Am J Obstet Gynecol, 2007. 196(2): p. 128.e1-6.

5. Nguyen, R.H., et al., Perceived stereotyping and seeking care for chronic vulvar pain. Pain Med, 2013. 14(10): p. $1461-7$.

6. Prendergast, S.A., Pelvic Floor Physical Therapy for Vulvodynia: A Clinician's Guide. Obstet Gynecol Clin North Am, 2017. 44(3): p. 509-522.

7. Stockdale, C.K. and H.W. Lawson, 2013 Vulvodynia Guideline update. J Low Genit Tract Dis, 2014. 18(2): p. 93-100.

8. Reed, B.D., et al., Treatment of vulvodynia with tricyclic antidepressants: efficacy and associated factors. J Low Genit Tract Dis, 2006. 10(4): p. 245-51.

9. Ayling, K. and J.M. Ussher, "If sex hurts, am I still a woman?" the subjective experience of vulvodynia in hetero-sexual women. Arch Sex Behav, 2008. 37(2): p. 294-304.

Page $11 / 15$ 
10. Xie, Y., et al., Economic burden and quality of life of vulvodynia in the United States. Curr Med Res Opin, 2012. 28(4): p. 6018.

11. Chisari, C. and J. Chilcot, The experience of pain severity and pain interference in vulvodynia patients: The role of cognitivebehavioural factors, psychological distress and fatigue. J Psychosom Res, 2017. 93: p. 83-89.

12. Muise, A., et al., Communal motivation in couples coping with vulvodynia: Sexual distress mediates associations with pain, depression, and anxiety. J Psychosom Res, 2018. 106: p. 34-40.

13. Paquet, M., et al., Daily Anxiety and Depressive Symptoms in Couples Coping With Vulvodynia: Associations With Women's Pain, Women's Sexual Function, and Both Partners' Sexual Distress. J Pain, 2018. 19(5): p. 552-561.

14. Reed, B.D., Vulvodynia: diagnosis and management. Am Fam Physician, 2006. 73(7): p. 1231-8.

15. Bergeron, S., et al., Vulvar vestibulitis syndrome: reliability of diagnosis and evaluation of current diagnostic criteria. Obstet Gynecol, 2001. 98(1): p. 45-51.

16. Wiegel, M., C. Meston, and R. Rosen, The female sexual function index (FSFI): cross-validation and development of clinical cutoff scores. J Sex Marital Ther, 2005. 31(1): p. 1-20.

17. Jiann, B.P., Pitfalls in using the female sexual function index. J Sex Med, 2012. 9(4): p. 1229.

18. Donders, G.G.G., G. Bellen, and K.S. Ruban, Abnormal vaginal microbioma is associated with severity of localized provoked vulvodynia. Role of aerobic vaginitis and Candida in the pathogenesis of vulvodynia. Eur J Clin Microbiol Infect Dis, 2018.

19. Harlow, B.L., et al., Recurrent Yeast Infections and Vulvodynia: Can We Believe Associations Based on Self-Reported Data? J Womens Health (Larchmt), 2017. 26(10): p. 1069-1076.

20. Friedrich, E.G., Jr., Vulvar vestibulitis syndrome. J Reprod Med, 1987. 32(2): p. 110-4.

21. Simon, J.A. and V.A. Lukas, Distressing Sexual Function at Midlife: Unmet Needs, Practical Diagnoses, and Available Treatments. Obstet Gynecol, 2017. 130(4): p. 889-905.

22. van Beekhuizen, H.J., J. Oost, and W.I. van der Meijden, Generalized unprovoked vulvodynia; A retrospective study on the efficacy of treatment with amitriptyline, gabapentin or pregabalin. Eur J Obstet Gynecol Reprod Biol, 2018. 220: p. 118-121.

23. McKay, M., Dysesthetic ("essential") vulvodynia. Treatment with amitriptyline. J Reprod Med, 1993. 38(1): p. 9-13.

24. Leo, R.J., A systematic review of the utility of anticonvulsant pharmacotherapy in the treatment of vulvodynia pain. J Sex Med, 2013. 10(8): p. 2000-8.

25. Spoelstra, S.K., C. Borg, and W.C. Weijmar Schultz, Anticonvulsant pharmacotherapy for generalized and localized vulvodynia: a critical review of the literature. J Psychosom Obstet Gynaecol, 2013. 34(3): p. 133-8.

26. Weinberger, J.M., et al., Female Sexual Dysfunction and the Placebo Effect: A Meta-analysis. Obstet Gynecol, 2018. 132(2): p. 453-458.

27. Goldstein, A.T., et al., Vulvodynia: Assessment and Treatment. J Sex Med, 2016. 13(4): p. 572-90.

28. Reed, B.D., et al., Pain at the vulvar vestibule: a web-based survey. J Low Genit Tract Dis, 2004. 8(1): p. 48-57.

29. Okura, Y., et al., Agreement between self-report questionnaires and medical record data was substantial for diabetes, hypertension, myocardial infarction and stroke but not for heart failure. Journal of Clinical Epidemiology, 2004. 57(10): p. 1096-1103.

\section{Tables}

Table 1. Sociodemographic characteristics of women with vulvodynia enrolled in the study $(n=144)$ 


\begin{tabular}{|c|c|}
\hline Variables & Frequency n (\%) \\
\hline Age (years, mean $\pm S D$ ) & $29( \pm 9.2)$ \\
\hline Caucasian ethinicity & $105(73 \%)$ \\
\hline \multicolumn{2}{|l|}{ Education } \\
\hline High school & $22(15.3 \%)$ \\
\hline College or undergraduate degree & $96(67 \%)$ \\
\hline Graduate school or professional training & $26(18.7 \%)$ \\
\hline \multicolumn{2}{|l|}{ Religion } \\
\hline Catholic & $68(46.5 \%)$ \\
\hline Other & $43(30.3 \%)$ \\
\hline None or atheist & $33(23.2 \%)$ \\
\hline BMI (mean \pm SD) & $22.6( \pm 4.8)$ \\
\hline \multicolumn{2}{|l|}{ Relationship status } \\
\hline No partner & $13(9.2 \%)$ \\
\hline Sporadic partner & $5(3.3 \%)$ \\
\hline Steady partner & $126(87.5 \%)$ \\
\hline \multicolumn{2}{|l|}{ Parity } \\
\hline Nulliparous & $100(69.4 \%)$ \\
\hline 1 & $14(9.7 \%)$ \\
\hline 2 or more & $30(20.9 \%)$ \\
\hline \multicolumn{2}{|l|}{ Miscarriage } \\
\hline Yes & $10(6.9 \%)$ \\
\hline \multicolumn{2}{|l|}{ Deliveries } \\
\hline None & $110(76.4 \%)$ \\
\hline Caesarion section & $22(15.3 \%)$ \\
\hline Vaginal birth & $12(8.3 \%)$ \\
\hline \multicolumn{2}{|l|}{ Previous pelvic surgery (other than cesarian) } \\
\hline Yes & $21.5(22.9 \%)$ \\
\hline \multicolumn{2}{|l|}{ Sedentary lifestyle } \\
\hline Yes & $58(40.3 \%)$ \\
\hline \multicolumn{2}{|l|}{ Associated health problem } \\
\hline Yes & $55(38.2 \%)$ \\
\hline \multicolumn{2}{|l|}{ Contraception method* } \\
\hline None & $2(1.4 \%)$ \\
\hline Hormonal & $61(41.3 \%)$ \\
\hline Condom & 33 (23\%) \\
\hline Other $^{(a)}$ & $60(14.1 \%)$ \\
\hline \multicolumn{2}{|l|}{ Tabaco use } \\
\hline Yes & $11(7.4 \%)$ \\
\hline
\end{tabular}

SD: Standard deviation; BMI: Body mass index; *More than one method possible; (a) diaphragm, tubal ligation, vasectomy, coitus interruptus, copper intrauterine device.

Table 2. Clinical characteristics of women with vulvodynia $(n=144)$ 


\begin{tabular}{|c|c|}
\hline \multicolumn{2}{|l|}{ Vulvovaginal signs and symptoms* } \\
\hline Vulvar erythema & $83(57.6 \%)$ \\
\hline Current vaginal discharge & $80(55.5 \%)$ \\
\hline Bad odor & $37(25.7 \%)$ \\
\hline Fissure & $30(20.8 \%)$ \\
\hline Oedema & $27(18.7 \%)$ \\
\hline Current vulva/vaginal burning & $117(81.2 \%)$ \\
\hline Itching & $52(36.1 \%)$ \\
\hline \multicolumn{2}{|l|}{ Patients seeking health care professionals ( $\left.\mathrm{n}=136^{* *}\right)$} \\
\hline \multicolumn{2}{|l|}{ None } \\
\hline 1 & $24(17.6 \%)$ \\
\hline 2 & $21(15.5 \%)$ \\
\hline 3 or more & $22(16.2 \%)$ \\
\hline & $69(50.7 \%)$ \\
\hline \multicolumn{2}{|l|}{ Type of health professional visited* $(n=136)$} \\
\hline 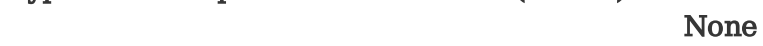 & $28(17.6 \%)$ \\
\hline Gynecologist & $104(76.5 \%)$ \\
\hline Psychiatrist & $32(23.5 \%)$ \\
\hline Physical therapist & $11(8.2 \%)$ \\
\hline Others $^{(a)}$ & $7(5.2 \%)$ \\
\hline \multicolumn{2}{|l|}{ Previous Diagnosis** $(\mathrm{n}=136)$} \\
\hline None & $67(49.2 \%)$ \\
\hline Low estrogen & $5(3.7 \%)$ \\
\hline Candidiasis & $21(15.5 \%)$ \\
\hline Non-organic ${ }^{(b)}$ & $3(2.2 \%)$ \\
\hline Vulvodynia & $40(29.4 \%)$ \\
\hline \multicolumn{2}{|l|}{ Vulvodynia setting } \\
\hline Primary & $61(42.4 \%)$ \\
\hline Secondary & $83(57.6 \%)$ \\
\hline \multicolumn{2}{|l|}{ Vulvodynia subtype } \\
\hline Localized provoked & $106(73.6 \%)$ \\
\hline Localized mixed & $8(5.5 \%)$ \\
\hline Generalized provoked & $12(8.4 \%)$ \\
\hline Generalized mixed & $18(12.5 \%)$ \\
\hline CST test according to NRS (mean \pm SD) & $5.1( \pm 3.3)$ \\
\hline Sexual pain on last 30 days (mean $\pm S D, n=132$ ) & $7.06( \pm 2.9)$ \\
\hline \multicolumn{2}{|l|}{ FSFI total score $(\mathrm{N}=126)$} \\
\hline$\leq 26.55$ & $118(93.6 \%)$ \\
\hline
\end{tabular}

VVD: Vulvodynia; SD: Standard deviation; *more than one answer possible; ** 8 missing data (did not know how to inform); (a)dermatologists, general practitioners, pain management therapist; (b)Psychological, allergy, hygiene or habits-related diagnosis; FSFI: Female Sexual Function Index; CST: cotton swab test for vulvodynia diagnosis; NRS: Numerical rating scale.

Table 3. Vulvar pain relief reported in previous treatment for vulvodynia 


\begin{tabular}{lcccc}
\hline \multicolumn{1}{c}{ Treatment or method used $(\mathrm{n}=110)$} & \multicolumn{3}{c}{ Vulvar pain relief } \\
\hline \multicolumn{1}{c}{$(\mathbf{N - \% )}$} & $\begin{array}{c}\text { No relief } \\
\mathbf{n}(\%)\end{array}$ & $\begin{array}{c}\text { Low } \\
\mathrm{n}(\%)\end{array}$ & $\begin{array}{c}\text { Mild } \\
\mathbf{n}(\%)\end{array}$ & $\begin{array}{c}\text { Strong } \\
\mathbf{n}(\%)\end{array}$ \\
\hline Lubricant (73-66.4\%) & $43(58.9)$ & $23(31.5)$ & $5(6.8)$ & $2(2.8)$ \\
\hline Topical anesthetic (40-36.4\%) & $4(10)$ & $21(52.5)$ & $10(25)$ & $5(12.5)$ \\
\hline Specific hygiene (37-33.6\%) & $26(70.3)$ & $8(21.6)$ & $3(8.1)$ & 0 \\
\hline Oral Antifungal (36-32.7\%) & $27(75)$ & $8(22.2)$ & $1(2.8)$ & - \\
\hline Oral Tricyclic antidepressant (34-31\%) & $11(32.3)$ & $16(47)$ & $5(14.7)$ & $2(6)$ \\
\hline Topical antifungal (28-25.5\%) & $25(89.2)$ & $1(3.6)$ & $1(3.6)$ & $1(3.6)$ \\
\hline Physical therapy for pelvic floor muscles (18-16.4\%) & $2(11.1)$ & $2(11.1)$ & $8(44.4)$ & $6(33.4)$ \\
\hline Psychotherapy approach (17-15.5\%) & $7(41.2)$ & $4(23.5)$ & $4(23.5)$ & $2(11.8)$ \\
\hline Other antidepressant (14-13\%) & $9(64.3)$ & $5(35.7)$ & - & - \\
\hline Topical hormone (11-10\%) & $3(27.3)$ & $5(45.4)$ & $2(18.2)$ & $1(9.1)$ \\
\hline Oral hormone therapy (8-7\%) & $6(75)$ & $1(12.5)$ & $1(12.5)$ & - \\
\hline Oral Gabapentin (6-5.2\%) & $2(33.3)$ & $2(33.3)$ & $1(16.6)$ & $1(16.6)$ \\
\hline Topical gabapentine (5-4.5\%) & $1(20)$ & $2(40)$ & $2(40)$ & 0 \\
\hline Low oxalate diet (5-4.3\%) & $3(60)$ & 0 & $1(20)$ & $1(20)$ \\
\hline Topical amitriptyline (3-2.6\%) & $2(66.7)$ & 0 & $1(33.3)$ & 0 \\
\hline
\end{tabular}

\section{Figures}

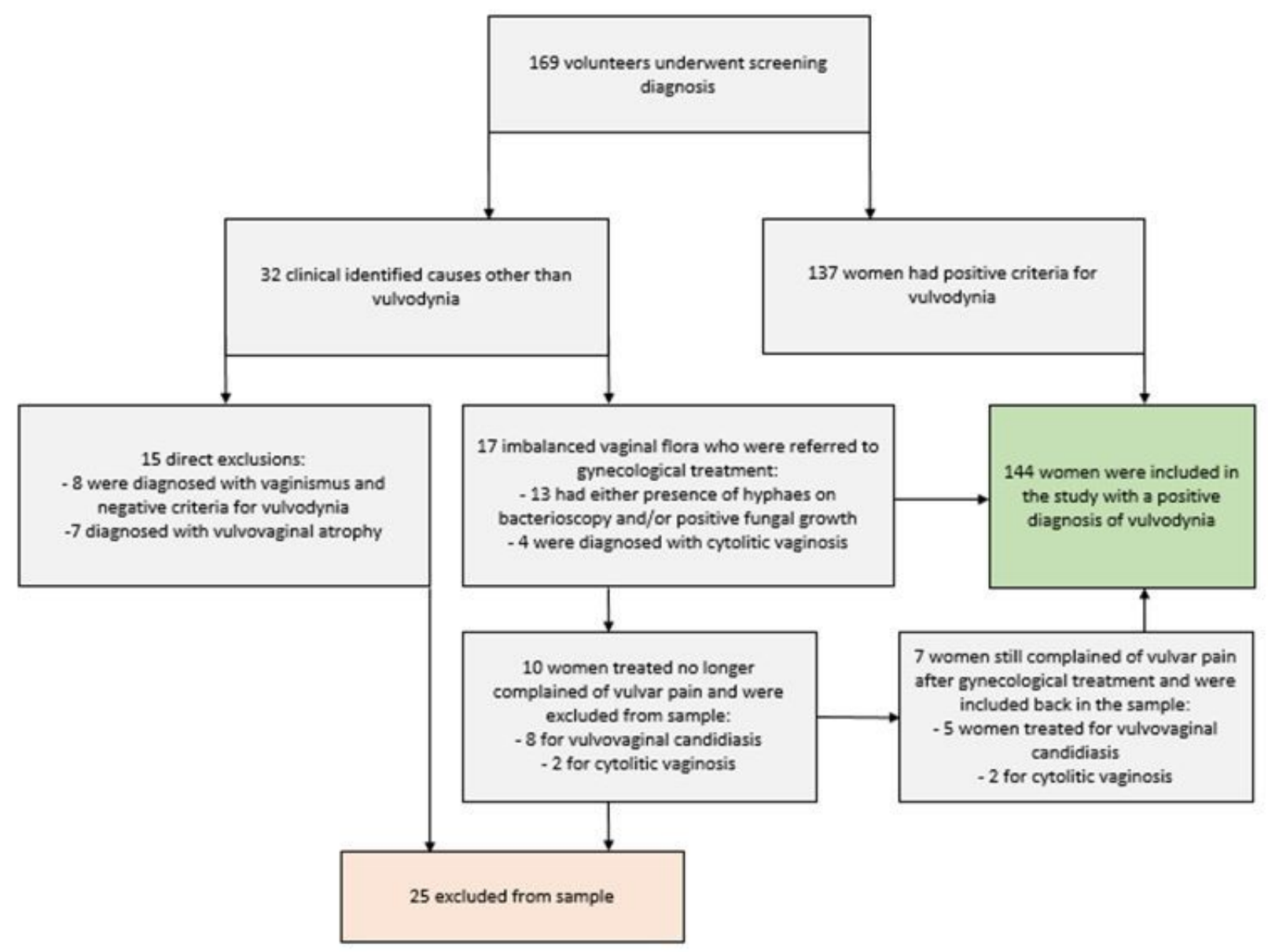

Figure 1

Flow chart of recruitment study. 